\title{
Production of the compatible solute a-D-glucosylglycerol by metabolically engineered Corynebacterium glutamicum
}

\author{
Benjamin Roenneke ${ }^{1,3}$, Natalie Rosenfeldt ${ }^{1}$, Sami M. Derya ${ }^{1}$, Jens F. Novak' ${ }^{1}$ Kay Marin ${ }^{1,4}$, Reinhard Krämer ${ }^{1}$ \\ and Gerd M. Seibold ${ }^{1,2^{*}}$ (B)
}

\begin{abstract}
Background: a-D-Glucosylglycerol (aGG) has beneficial functions as a moisturizing agent in cosmetics and potential as a health food material, and therapeutic agent. aGG serves as compatible solute in various halotolerant cyanobacteria such as Synechocystis sp. PCC 6803, which synthesizes aGG in a two-step reaction: The enzymatic condensation of ADP-glucose and glycerol 3-phosphate by GG-phosphate synthase (GGPS) is followed by the dephosphorylation of the intermediate by the GG-phosphate phosphatase (GGPP). The Gram-positive Corynebacterium glutamicum, an industrial workhorse for amino acid production, does not utilize aGG as a substrate and was therefore chosen for the development of a heterologous microbial production platform for aGG.
\end{abstract}

Results: Plasmid-bound expression of ggpS and ggpP from Synechocystis sp. PCC 6803 enabled aGG synthesis exclusively in osmotically stressed cells of C. glutamicum (pEKEx2-ggpSP), which is probably due to the unique intrinsic control mechanism of GGPS activity in response to intracellular ion concentrations. C. glutamicum was then engineered to optimize precursor supply for aGG production: The precursor for aGG synthesis ADP-glucose gets metabolized by both the glgA encoded glycogen synthase and the otsA encoded trehalose-6-phosphate synthase. Upon deletion of both genes the aGG concentration in culture supernatants was increased from $0.5 \mathrm{mM}$ in C. glutamicum (pEKEx3ggpSP) to $2.9 \mathrm{mM}$ in C. glutamicum $\triangle$ otsA IMglgA (pEKEx3-ggpSP). Upon nitrogen limitation, which inhibits synthesis of amino acids as compatible solutes, C. glutamicum $\triangle$ otsA IMglgA (pEKEx3-ggpSP) produced more than 10 mM aGG (about $2 \mathrm{~g} \mathrm{~L}^{-1}$ ).

Conclusions: Corynebacterium glutamicum can be engineered as efficient platform for the production of the compatible solute aGG. Redirection of carbon flux towards aGG synthesis by elimination of the competing pathways for glycogen and trehalose synthesis as well as optimization of nitrogen supply is an efficient strategy to further optimize production of aGG.

Keywords: Corynebacterium glutamicum, Trehalose, Compatible solute, Glycogen, a-D-Glucosylglycerol

\footnotetext{
*Correspondence: gerd.seibold@uni-ulm.de

${ }^{2}$ Institute of Microbiology and Biotechnology, Ulm University,

Albert-Einstein Allee 11,89081 Ulm, Germany

Full list of author information is available at the end of the article
} 


\section{Background}

Accumulation of compatible solutes is required for the acclimation of organisms to adverse environmental conditions with increasing external osmolality, particularly to salt and drought stress $[1,2]$. Compatible solutes are low molecular weight compounds, which can be accumulated in the cytoplasm to molar concentrations without negatively affecting cellular functions and metabolism [3]. Additionally, these solutes are able to protect cellular macromolecules directly by stabilizing their hydration shell [4-6]. When accumulated in response to hyperosmotic stress compatible solutes increase the internal osmolality, which safeguards water uptake and constant turgor pressure $[1,7]$. The accumulation of compatible solutes is achieved by either de novo synthesis or uptake. Both processes are part of the immediate response to osmotic stress and comprise the direct activation of preformed enzymes [8].

Currently many compatible solutes such as ectoine, hydroxyectoine, and trehalose are widely used as moisturizing agents in cosmetics $[9,10]$, stabilizing agents for enzyme preparations, and/or as xeroprotectants for the storage of biomaterials and mammalian cells [11-13]. The compatible solute $\alpha$-D-glucosylglycerol $(\alpha G G)$ has various beneficial functions and a large potential as a health food material, therapeutic agent, and for enzyme stabilization [14, 15]. $\alpha$ GG serves as a compatible solute in various halotolerant cyanobacteria such as Synechocystis sp. PCC $6803[16,17]$. In this organism $\alpha \mathrm{GG}$ is synthesized in a two-step reaction in which the enzymatic condensation of ADP-glucose and glycerol 3-phosphate by GG-phosphate synthase (GGPS) is followed by the dephosphorylation of the intermediate by the GG-phosphate phosphatase (GGPP) $[18,19]$. The mechanism for the control of $\alpha$ GG synthesis in Synechocystis sp. PCC 6803 was recently described. In the absence of salt stress, the enzyme GgpS is bound to DNA in inactive form. Upon an increase in the extracellular salt concentration, $\mathrm{Na}^{+}$enters the cell. The rise in the cytoplasmic $\mathrm{Na}^{+}$concentration interferes with the binding of the GgpS protein to DNA leading to the detachment of the enzyme. As a result, the enzyme is activated and catalyzes the synthesis of $\alpha$-glucosylglycerol-phosphate [20]. Enzymatic syntheses using different glycosyltransferases (e.g. sucrose phosphorylase from Leuconostoc mesenteroides, $\alpha$-glucosidase from Halomonas sp. strain H11, or amylosucrase from Methylobacillus flagellates) were developed [21-23] and are currently applied for the industrial production of $\alpha$ GG. These enzymatic processes depend on pure substrates [22], as impurities lead to formation of unwanted by-products or inhibition of the process. In difference the use of microbes as biocatalyst allows feedstock flexibility, as the cells can be genetically engineered to efficiently generate required precursors [24, 25]. Recently, Synechocystis sp. PCC 6803 was optimized for $\alpha$ GG production [26]. For this purpose, $g g p R$ as well as $g g t C$ and $g g t D$ were disrupted, which encode the transcriptional repressor GgpR of ggpS and ggpP and the components of the $\alpha \mathrm{GG}$ uptake system [17], respectively. The resulting Synechocystis sp. PCC 6803 strain produced extracellular $\alpha \mathrm{GG}$, however, it required first hyperosmotic stress for intracellular $\alpha$ GG synthesis via GGPS and GGPP and then a switch to hypoosmotic conditions to trigger $\alpha \mathrm{GG}$ secretion via mechanosensitive channels in the culture broth [26]. Engineering of non-natively $\alpha$ GG synthesizing bacteria towards production of this compatible solute has hitherto not been described [15].

The Gram-positive soil bacterium Corynebacterium glutamicum, a well-established industrial workhorse for amino acid production [27, 28], has been genetically engineered into a suitable platform for the production of various bulk and commodity chemicals [25, 29]. Recently, C. glutamicum has been genetically engineered towards efficient production of the compatible solutes trehalose and ectoine [30-34]. C. glutamicum itself is highly osmotolerant and accumulates by synthesis or uptake large amounts of compatible solutes in response to hyperosmotic stress [35]. When cultivated in minimal medium $C$. glutamicum wild type mainly synthesizes the amino acid proline as compatible solute in response to hyperosmotic stress. When nitrogen is limiting trehalose is synthesized as compatible solute via the OtsAB and the TreYZ pathways $[36,37]$.

In this communication, we describe the genetic engineering of C. glutamicum for the production of $\alpha \mathrm{GG}$. This organism transiently accumulates in the course of cultivations with sugars as substrate large amounts of glycogen [36, 38-42]. As glycogen synthesis depends on the formation of the intermediate ADP-glucose [41], which is also the precursor for $\alpha \mathrm{GG}$ synthesis, it seemed likely that $C$. glutamicum might be suited as $\alpha$ GG production organism. In addition, formation of the second $\alpha \mathrm{GG}$ precursor glycerol-3-phosphate has been observed in $C$. glutamicum cells [43]. We overexpressed ggpS and ggpP from Synechocystis sp. PCC 6803 in C. glutamicum and thus enabled $\alpha$ GG synthesis in osmotically stressed cells of the derived strain. We further analyzed molecular targets to optimize supply of the $\alpha$ GG precursor ADP-glucose and optimize cultivation conditions. By this means we here describe for the first time a strategy for efficient microbial production of $\alpha \mathrm{GG}$.

\section{Methods}

Bacterial strains, media, and culture conditions

Bacteria and plasmids used in this study are listed in Table 1. E. coli and all pre-cultures of C. glutamicum 
Table 1 Strains and plasmids used in this study

\begin{tabular}{|c|c|c|}
\hline Strain/plasmid & Relevant characteristics & References/source \\
\hline \multicolumn{3}{|l|}{ E. coli strains } \\
\hline $\mathrm{DH} 5 \mathrm{a}$ & 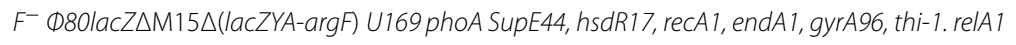 & [68] \\
\hline BL21(DE3) & ompThsdS $S_{B}\left(r_{B}^{-} m_{B}^{-}\right) g a l d c m(D E 3)$ & [69] \\
\hline \multicolumn{3}{|l|}{ C. glutamicum strains } \\
\hline WT & Wild-type strain ATCC 13032 & American type culture collection \\
\hline$\triangle o t s A$ & C. glutamicum WT with deletion of otsA (cg2907) & {$[37]$} \\
\hline IMglgA & C. glutamicum WT with inactivation of glgA (cg1268) & This work \\
\hline$\triangle$ otsA IMglgA & C. glutamicum WT with deletion of ots $A$ and inactivation of $g / g A$ & This work \\
\hline$\triangle o t s A \Delta t r e Y \Delta t r e s$ & C. glutamicum WT with deletion of otsA (cg2907), treY (cg2323), and tres (cg2529) & {$[37]$} \\
\hline \multicolumn{3}{|l|}{ Plasmids } \\
\hline pEKEX2 & 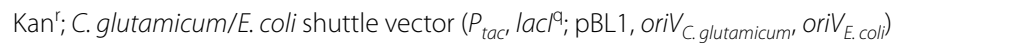 & [70] \\
\hline pEKEx2-GgpP & Derived from pEKEx2, for expression of ggpP in C. glutamicum & This work \\
\hline pEKEx2-GgpPS & Derived from pEKEx2, for expression of ggpP and ggpS in C. glutamicum & This work \\
\hline pEKEx3 & 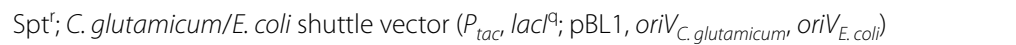 & [71] \\
\hline pEKEX3-GgpPS & Derived from pEKEx3, for expression of ggpP and ggpS in C. glutamicum & This work \\
\hline pDrive & Kann $^{r}$, Ampr; E. coli cloning vector (lacZa, orif1, ori-pUC) & Qiagen, Hilden, Germany \\
\hline pDrive-IMglgA & Derived from pDrive, vector carrying internal region of $g \lg A(\mathrm{cg} 1268)$ for inactivation & This work \\
\hline PASK-IBA3 & Overexpression vector (STREP Tagll); Ampr & IBA GmbH, Göttingen, Germany \\
\hline pASK-IBA3-otsA & Derived from pASK-IBA3, for regulated expression of otsA (cg2907) of C. glutamicum & This work \\
\hline
\end{tabular}

were grown aerobically in TY complex medium [44] at 37 and $30{ }^{\circ} \mathrm{C}$, respectively, as $50-\mathrm{mL}$ cultures in $500-\mathrm{mL}$ baffled Erlenmeyer flasks on a rotary shaker at $120 \mathrm{rpm}$. For the main cultures of C. glutamicum, cells of an overnight pre-culture were washed twice with $0.9 \%(\mathrm{w} / \mathrm{v})$ $\mathrm{NaCl}$ and then inoculated into CGC minimal medium [45] containing glucose or sucrose as carbon sources as indicated in "Results" section. When appropriate, kanamycin $\left(25\right.$ or $\left.50 \mu \mathrm{g} \mathrm{mL}^{-1}\right)$, spectinomycin $\left(30 \mu \mathrm{g} \mathrm{mL}{ }^{-1}\right)$, carbenicillin $\left(30 \mu \mathrm{g} \mathrm{mL} \mathrm{m}^{-1}\right)$, and/or isopropyl- $\beta$-Dthiogalactopyranoside (IPTG, $250 \mu \mathrm{M}$ ) were added to the media. For growth experiments in presence of nitrogenlimitation $\mathrm{CgC}$-medium without ammonia and varying amounts of urea were used as indicated in "Results" section; salt stress was induced by direct addition of $\mathrm{NaCl}$ to cultivation vessels to final concentrations indicated in "Results" section. Growth of E. coli and of C. glutamicum was followed by measuring the optical density at $600 \mathrm{~nm}$ $\left(\mathrm{OD}_{600}\right)$. For analyses of $\alpha \mathrm{GG}$ production in bioreactors growth experiments were performed aerobically at $30{ }^{\circ} \mathrm{C}$ as $1 \mathrm{~L}$ cultures in $1.5 \mathrm{~L}$ jars in a Biostat B fermentation system from Braun essentially as described before [40]. The $\mathrm{pH}$ was maintained at 7.0 by online measurement using a standard $\mathrm{pH}$ electrode (Mettler Toledo) and the addition of $4 \mathrm{M} \mathrm{KOH}$ and $15 \%(\mathrm{v} / \mathrm{v}) \mathrm{H}_{2} \mathrm{SO}_{4}$. Dissolved oxygen was measured online by use of a polarimetric oxygen electrode (Mettler Toledo) and it was adjusted to 30\% of saturation in a cascade by stirring at 100 to $1200 \mathrm{rpm}$ and aerating with $3 \mathrm{~L}$ of air per minute.

\section{Analysis of product and substrate concentrations}

$\alpha G G$ concentrations in culture supernatants were analyzed using GC as recently described [20]. In the course of fermentation experiments substrate and product (sucrose and $\alpha \mathrm{GG}$ ) concentrations in the culture supernatants were analyzed using a Hitachi HPLC system equipped with a L2130 gradient pump, a L2200 column oven, an L2350 auto sampler, a L2490 RI detector, and a Nucleo Sugar 810Ca (300/7.8) column plus Nucleogel Sugar $810 \mathrm{Ca}(30 / 4)$ precolumn (Macherey \& Nagel). The mobile phase was $0.005 \mathrm{M} \mathrm{CaCl}_{2}$ in water with a flow rate of $0.7 \mathrm{~mL} \mathrm{~min}^{-1}$, the column temperature was $60{ }^{\circ} \mathrm{C}$ and the sample volume $5 \mu \mathrm{L}$. Calibration was performed using a serial dilution of a solution containing $50 \mathrm{mM}$ sucrose (Sigma-Aldrich) and $50 \mathrm{mM} \alpha \mathrm{GG}$ (Bitop AG). The dry weight of C. glutamicum in the course of cultivations was calculated according to the $\mathrm{OD}_{600}$; as described recently [40].

For the analysis of intracellular $\alpha \mathrm{GG}$ concentrations 2-mL samples were taken from the cultures and immediately centrifuged $\left(2 \mathrm{~min}, 13,000 \mathrm{rpm}, 4^{\circ} \mathrm{C}\right)$. The resulting cell pellets were washed twice with fresh isoosmotic minimal medium to remove external $\alpha$ GG. The obtained pellets were suspended in $1 \mathrm{~mL}$ of methanol and incubated for $20 \mathrm{~min}$ at $70{ }^{\circ} \mathrm{C}$ to extract low molecular mass compounds. The dry residue was treated with $0.5 \mathrm{~mL}$ chloroform and $1 \mathrm{~mL}$ water to remove membranes, pigments and soluble proteins. After centrifugation, the aqueous phase was taken and dried in a vacuum concentrator. In 
order to remove high concentrations of salts and add the internal standard ribitol, the dry residue was dissolved in $80 \mu \mathrm{L}$ absolute ethanol containing $200 \mu \mathrm{g} \mathrm{mL} \mathrm{m}^{-1}$ ribitol. After centrifugation, the liquid phase was transferred into a new vial, dried and stored at room temperature until analysis of the $\alpha \mathrm{GG}$ concentrations by GC was performed as described [46].

\section{DNA isolation, transfer, and manipulations}

Standard procedures were employed for plasmid isolation, cloning, and transformation of E. coli DH5 $\alpha$, as well as for electrophoresis [44]. Transformation of C. glutamicum was performed by electroporation using the methods described in Ref. [47], the recombinant strains were selected on LB-BHIS agar plates containing kanamycin $\left(25 \mu \mathrm{g} \mathrm{mL}^{-1}\right)$ and/or spectinomycin $\left(30 \mu \mathrm{g} \mathrm{mL}^{-1}\right)$. Electroporation of $E$. coli was performed using the methods mentioned in the Ref. [48]. All enzymes used were obtained from New England Biolabs and used according to the instructions of the manufacturer. PCR experiments were performed in a Tprofessional thermocycler (Biometra). Deoxynucleotide triphosphates were obtained from Bio-Budget, oligonucleotides (primers) from Eurofins MWG Operon (Table 2). Cycling times and temperatures were chosen according to fragment length and primer constitution. PCR products were separated on agarose gels and purified using the Nucleospin extract II kit (Macherey \& Nagel). All cloned fragments were verified by sequencing (GATC Biotech AG).

\section{Heterologous expression of ggpS and $g g p P$ in $C$. glutamicum}

For IPTG-inducible heterologous expression of ggpS and ggpP the vectors pEKEx2 and pEKEx3 were used. The ggpP gene was amplified via PCR from genomic DNA of Synechocystis sp. PCC6803 using the oligonucleotide primers ggpP_5'_cgl and ggpP_3'_cgl. Using the PCRgenerated KpnI and EcoRI restriction sites of PCR product, the $1564 \mathrm{bp}$ PCR product was ligated into pEKEx2 and transformed into E. coli. The resulting plasmid pEKEx2-ggpP was isolated from E. coli. The ggpS gene was amplified including the strep-tag coding sequence from the plasmid pASK-IBA-3-ggpS-strep using the oligonucleotide primers ggpS_5'_cgl and ggpS_3'_cgl. Using the PCR-generated PstI and BamHI restriction sites of PCR product, the $1570 \mathrm{bp}$ PCR product was ligated into pEKEx2-ggpS and transformed into E. coli. The resulting plasmid pEKEx2-ggpSP was isolated from E. coli and the nucleotide sequence controlled by sequencing (GATC Biotech). For heterologous expression of ggpS and ggpP in kanamycin-resistant $C$. glutamicum integration mutants the plasmid pEKEx3-ggpSP was constructed. For this purpose, the 5013 bp fragment carrying ggpP and
ggpS was excised from pEKEx2-ggpSP using XhoI and PstI and cloned into pEKEx3 cut with the same enzymes, and transformed into $E$. coli. The resulting plasmid pEKEx3-ggpSP was isolated from E. coli and the nucleotide sequence controlled by sequencing.

\section{Inactivation of $g l g A$ in C. glutamicum strains}

Inactivation of the chromosomal $g l g A$ gene (orf cg1268) in C. glutamicum WT and C. glutamicum $\triangle$ ots $A$ was performed essentially as described for the inactivation of the pgm gene [38], using the plasmid pDrive-IMglgA. This plasmid was constructed by PCR-amplification of a DNA fragment covering nucleotides $85-636$ of the annotated $\operatorname{glg} A$ gene, using primers glgA-IM-for and glgA-IM-rev (Table 1). The 551 bp PCR product was directly cloned into the TA-cloning vector pDrive (Qiagen) according to the manufacturer's instructions and the resulting vector pDrive-IMglgA transformed into E. coli DH5 $\alpha$. After isolation of the recombinant plasmid, it was electroporated into C. glutamicum WT and C. glutamicum $\triangle$ otsA. Integration of pDrive-IMglgA at the genomic $g \lg A$ locus in C. glutamicum and thus inactivation of the $g l g A$ gene was confirmed by PCR using primers glgA-IM-rev and M13-FP resulting in a specific 683 bp product for C. glutamicum IMglgA and C. glutamicum $\triangle$ otsA IMglgA.

\section{Overproduction, purification, and activity measurements for the trehalose 6-phosphate synthase OtsA from $C$. glutamicum}

For heterologous expression in E. coli, the otsA gene of C. glutamicum was amplified from genomic DNA by PCR using the primers otsA-fw and otsA-rv (Table 1) and the resulting fragment cloned into the vector pASKIBA3 (IBA GmbH, Göttingen Germany), according to the supplier's manual. Expression of otsA-strep was induced by the addition of anhydrotetracycline $\left(2 \mu \mathrm{g} \mathrm{mL}{ }^{-1}\right.$ at an OD600 of 1, and cells were harvested 6 h later by centrifugation at $5000 \times g$ for $5 \mathrm{~min}$. The cells were suspended in wash buffer $(100 \mathrm{mM}$ Tris- $\mathrm{HCl}, \mathrm{pH}$. 8.0, and $150 \mathrm{mM}$ $\mathrm{NaCl}$ ) and disrupted by passing three times through a French pressure cell press (SLM-AMINCO) at 18,000 psi. After centrifugation at $15,000 \times g$ for $20 \mathrm{~min}$, the supernatant was subjected to Strep-tag purification (IBA GmbH) according to the supplier's manual. The purified OtsA enzyme was frozen in liquid nitrogen and stored at -80 ${ }^{\circ} \mathrm{C}$ until further use.

The activity of purified OtsA was determined using an assay similar to the assay recently described for the determination of GgpS activity [20]. In detail, $0.2 \mu \mathrm{g}$ of purified OtsA were incubated in a total volume of $80 \mu \mathrm{L}$ at $30{ }^{\circ} \mathrm{C}$ for $10 \mathrm{~min}$ in assay buffer $(10 \mathrm{mM}$ TRIS-maleate, $5 \mathrm{mM} \mathrm{MgCl}, 0.05 \mathrm{mM}$ EDTA, pH 7.5) in presence of $3 \mathrm{mM}$ of UDP-glucose or ADP-glucose and $3 \mathrm{mM}$ of 
Table 2 Oligonucleotides used in this study

\begin{tabular}{lll}
\hline Oligonucleotide & Sequence & Purpose \\
\hline ggps_5'_cgl & 5'-GCCTGCAGAGGAGAGGGCAAAAAATGAATTCATCCC-3' $^{\prime}$ & pEKEx2-GgpPS \\
ggpS_3'_cgl & 5'-GCGGATCCTCACTTCACAGGTCAAGCTTAT-3' & pEKEx2-GgpPS \\
ggpP_5'_cgl & 5'-GCGGTACCAGGAGGAGGCTAATAATGGTATTACACCAACAAC-3' & pEKEx2-GgpP \\
ggpP_3'_cgl & 5'-GCGAATTCTTGCTAGTGGTGGTGGTGGTGGTGCTGGGAAAAA TGGACTCTTCGG & pEKEx2-GgpP \\
& CGCTGGGCCGCCTGCT-3' & pASK-IBA3-otsA \\
otsA-for & 5'-GCTCTAGAGCATGGATGATTCCAATAGCTT-3' & pASK-IBA3-otsA \\
otsA-rev & 5'-GCCCTCGAGCGGTGAGTTTTCTCCCGACTGTG-3' & pDrive-IMglgA \\
glgA-IM-for & 5'-TGATGCGGCCGACAAATG-3' & pDrive-IMglgA, analysis of \\
glgA-IM-rev & 5'-TGCGTGAGATCGCTGAAG-3' & glgA inactivation \\
M13-FP & & analysis of glgA inactivation \\
\hline
\end{tabular}

glucose-6-phosphate as substrates. The reaction was stopped by incubation for $10 \mathrm{~min}$ at $95{ }^{\circ} \mathrm{C}$ and formed trehalose-6-phosphate was then dephosphorylated by addition of $1 \mathrm{U}$ alkaline phosphatase (FastAP, Fermentas, St. Leon-Roth, Germany) and incubation for $30 \mathrm{~min}$ at $30{ }^{\circ} \mathrm{C}$. Sample preparation for gas chromatography and analyses of trehalose concentrations by split injection capillary gas chromatography using xylitol as internal and trehalose as external standard were performed as described by Novak et al. [20] and Wolf et al. [37], respectively.

\section{Protein analysis}

Protein concentrations were determined using the RotiNanoquant kit (Roth) with bovine serum albumin as the standard. SDS-PAGE was performed according to Laemmli [49]. Standard loading buffer $(4 \times)$ contained $8 \%$ (w/v) SDS, 20\% (v/v) glycerol, $10 \mathrm{mM}$ EDTA, $100 \mathrm{mM}$ Tris/HCl, pH 6.8, 2\% (v/v) $\beta$-mercaptoethanol, and $1 \mathrm{mg}$ bromphenol blue $\mathrm{mL}^{-1}$. Western blot experiments for the detection of His-tagged GgpP was performed as described for GlgX-His [39]. Detection of the Streptavidin-tagged GgpS protein and determination of GgpS activity in cell free extracts of C. glutamicum strains were performed as recently described [20].

\section{Results \\ $\mathrm{NaCl}$-triggerd aGG synthesis in C. glutamicum (pEKEx2-ggpSP)}

The compatible solute $\alpha \mathrm{GG}$ is synthesized in Synechocystis sp. PCC 6803 in a two-step reaction in which the enzymatic condensation of ADP-glucose and glycerol 3-phosphate by GG-phosphate synthase (GGPS) is followed by the dephosphorylation of the intermediate by the GG-phosphate phosphatase (GGPP). The genes ggpS and ggpP from Synechocystis sp. PCC 6803 were cloned into the expression plasmid pEKEx2 and the resulting plasmid was introduced into C. glutamicum. Presence of the plasmid encoded enzymes in extracts of $C$. glutamicum (pEKEx2-ggpSP) was shown by western blot analysis using antibodies raised against the C-terminal His-Tag of GgpP and the C-terminal Strep-Tag of GgpS (Fig. 1a), and a GgpS activty of $9.52 \pm 2.06 \mu \mathrm{mol} \mathrm{min}{ }^{-1}$ (mg protein $)^{-1}$ was observed in cell free extracts of $C$. glutamicum (pEKEx2-ggpSP). However, no formation of $\alpha G G$ was observed in samples from the culture supernatant of C. glutamicum (pEKEx2-ggpSP) during cultivation in minimal medium with $1 \%(\mathrm{w} / \mathrm{v})$ sucrose (Fig. 1b, c). GgpS from Synechocystis sp. PCC 6803 underlies a post-translational regulatory mechanism, which is based on salt-dependent electrostatic interaction of the protein with the negatively charged backbone of polyanions, e.g. DNA and RNA) [20]. This mode of activity control is probably conserved even when the gene is heterologously expressed in C. glutamicum. Indeed the GgpS activity in extracts of C. glutamicum (pEKEx2-ggpSP) increased to $27.64 \pm 1.3 \mu \mathrm{mol} \mathrm{min}{ }^{-1}(\mathrm{mg} \text { protein })^{-1}$ when $200 \mathrm{mM}$ $\mathrm{NaCl}$ was present in the assay buffer. To activate the GgpS in C. glutamicum (pEKEx3-ggpSP) the cells were stressed during cultivation by addition of $\mathrm{NaCl}$ (final concentration $750 \mathrm{mM}$ ). Accumulation of $\alpha \mathrm{GG}$ was obserevd exclusively in the culture supernatants sampled after the addition of $\mathrm{NaCl}$ (Fig. 1b, c), after $25 \mathrm{~h}$ cultivation $0.62 \pm 0.07 \mathrm{mM} \alpha \mathrm{GG}$ was formed. More detailed experiments with C. glutamicum (pEKEx2-ggpSP) showed the dependence of externally accumulated $\alpha G G$ on the $\mathrm{NaCl}$ concentration during cultivation, an increase of the external $\mathrm{NaCl}$ concentrations caused increased formation of $\alpha$ GG (Fig. 2a). No variations of GgpS amounts were detected in western blot analyses with an antibody against the C-terminal Strep-Tag of GgpS, when cells extracts of $C$. glutamicum (pEKEx2-ggpSP) from the cultivations at different amounts of $\mathrm{NaCl}$ were analyzed (Fig. 2b). 


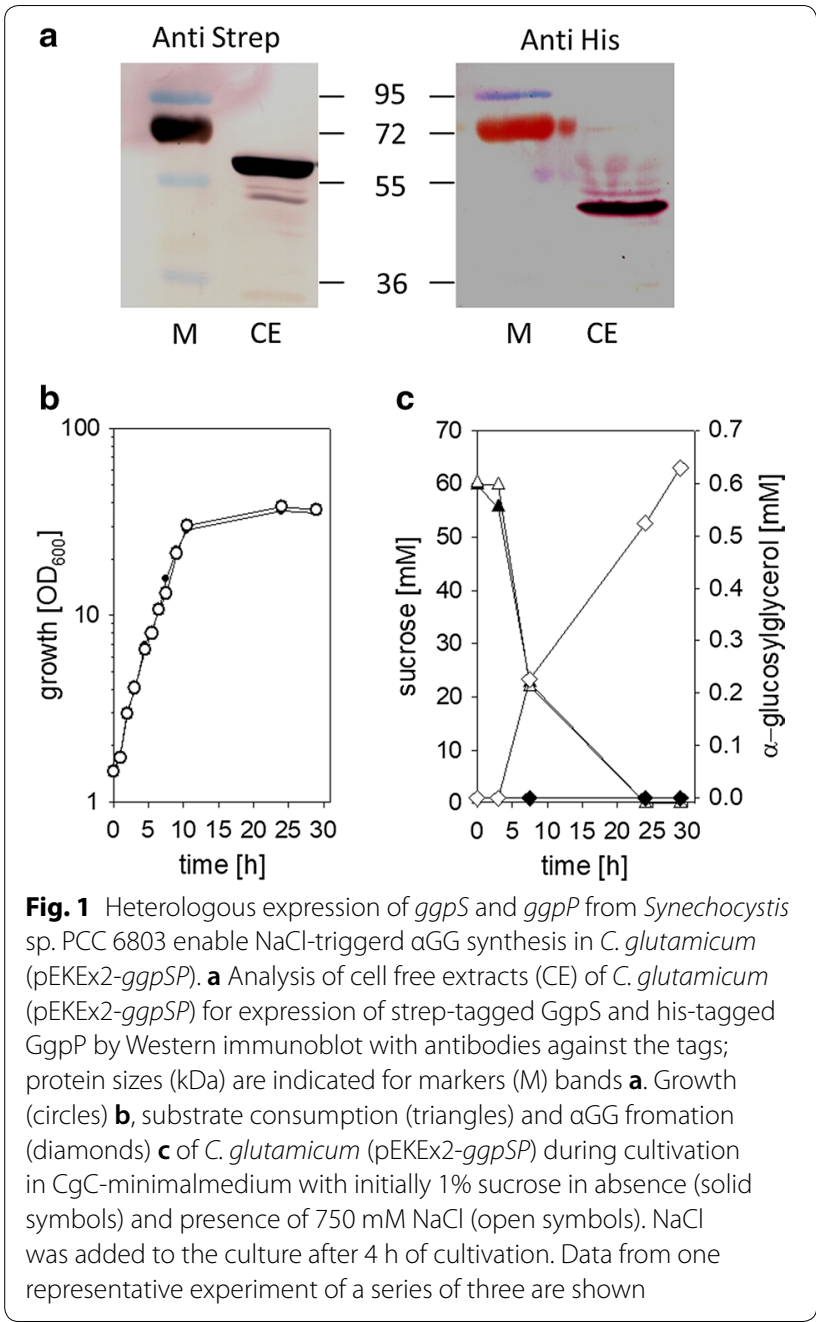

$\mathrm{NaCl}$-dependent accumulation of $\alpha \mathrm{GG}$ in supernatants of C. glutamicum (pEKEx2-ggpSP) depends on both intracellular synthesis of $\alpha \mathrm{GG}$ and $\alpha \mathrm{GG}$ export. Intracellular $\alpha \mathrm{GG}$ was analysed in cells of C. glutamicum (pEKEx2-ggpSP) in samples taken from cultivations $6 \mathrm{~h}$ after the addition of $600 \mathrm{mM} \mathrm{NaCl}$ and from untreated cultures. Intracellular $\alpha \mathrm{GG}$ concentration of about $12 \mathrm{mM}$ and $450 \mathrm{mM}$ were measured for cells of C. glutamicum (pEKEx2-ggpSP) from untreated and $\mathrm{NaCl}$-shocked cultivations, respectively. This results indicates that in absence of $\mathrm{NaCl}$-stress the $\mathrm{GgpS}$ activity was low, whereas the addition of $\mathrm{NaCl}$ to the medium indeed activated intracellular $\alpha G G$ synthesis by the GgpS.

Taken together these data show, that upon $\mathrm{NaCl}-$ induced stress GgpS is activated in C. glutamicum (pEKEx2-ggpSP) similar as in Synechocystis sp. PCC 6803 leading to the synthesis of $\alpha \mathrm{GG}$, which is then exported to the culture medium different from the situation in Synechocystis sp. PCC 6803.

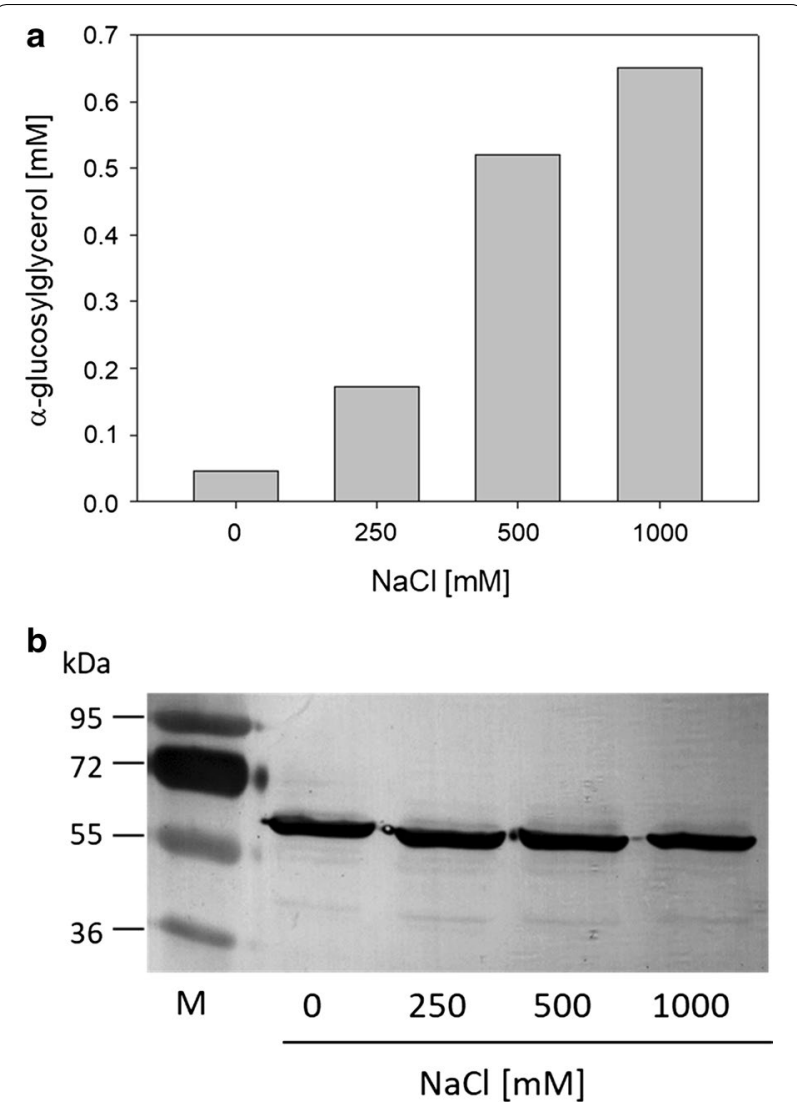

Fig. 2 Analysis of the $\mathrm{NaCl}$ dependency of aGG synthesis in C. glutamicum (pEKEx2-ggpSP). aGG concentrations in culture supernatants after $24 \mathrm{~h}$ cultivation of C. glutamicum (pEKEx2-ggpSP) in minimal medium with initially $1 \%$ sucrose as sole carbon source $\mathbf{a}$. $\mathrm{NaCl}$ was added in indicated concentrations after $4 \mathrm{~h}$ of cultivation. Analysis of cell free extracts (CE) of C. glutamicum (pEKEx2-ggpSP) cultivated at different $\mathrm{NaCl}$ concentrations for expression of strep-tagged GgpS by Western immunoblot with antibodies against the tag $\mathbf{b}$; protein sizes $(\mathrm{kDa})$ are indicated for markers $(\mathrm{M})$ bands

\section{Optimization of precursor supply for efficient aGG synthesis in C. glutamicum}

GgpS of Synechocystis sp. PCC 6803 requires ADP-glucose as a substrate and does not utilize alternative activated sugars such as UDP-glucose [50]. The precursor for $\alpha G G$ synthesis ADP-glucose is a key intermediate of glycogen synthesis. In C. glutamicum ADP-glucose is formed by the $g l g C$ encoded ADP-glucosepyrophosphoralyse and used for the elongation of glucan chains by the $g l g A$ encoded glycogen synthase $[40,41]$. The genes $g l g C$ and $\operatorname{glg} A$, are situated adjacent on the genome sequence of C. glutamicum, separated by $153 \mathrm{bp}$, and are divergently transcribed [51]. Thus inactivation of $g l g A$ by insertion mutagenesis is possible in C. glutamicum without affecting $g l g C$ [36]. The gene $g l g A$ was inactivated by insertion of the vector pDrive, the resulting strain $C$. glutamicum IMglgAdid not accumulate glycogen during 
cultivation in minimal medium with either $1 \%(\mathrm{w} / \mathrm{v})$ glucose or $1 \%(\mathrm{w} / \mathrm{v})$ sucrose as substrate (data not shown). For heterologous expression of ggpS and ggpP the plasmid pEKEx3-ggpSP was introduced into C. glutamicum IMglgA. During cultivation in minimal medium with $2 \%$ $(\mathrm{w} / \mathrm{v})$ sucrose and $750 \mathrm{mM} \mathrm{NaCl}$ the resulting strain $C$. glutamicum IMglgA (pEKEx3-ggpSP) accumulated within $24 \mathrm{~h}$ of cultivation $3.23 \pm 0.15 \mathrm{mM} \alpha \mathrm{GG}$ in its supernatant, which is about 6 times more than the amount of $0.57 \pm 0.06 \mathrm{mM} \alpha \mathrm{GG}$ observed for the parental strain $C$. glutamicum (pEKEx3-ggpSP) (Table 3). The initially provided carbon source sucrose was completely consumed by both strains, C. glutamicum IMglgA (pEKEx3-ggpSP) formed about $2.3 \mathrm{~g} \mathrm{~L}^{-1}$ less biomass than C. glutamicum (pEKEx3-ggpSP).

Besides for glycogen synthesis ADP-glucose is also used in some bacteria such as Propionibacterium freudenreichii for trehalose synthesis. Usually the trehalose-6-phosphate synthase OtsA uses UDP-glucose as substrate [52], but the enzyme from $P$. freudenreichii prefers ADP-glucose over UDP-glucose [53]. In C. glutamicum OtsA contributes to trehalose synthesis [36, 37], the substrate spectrum of OtsA from C. glutamicum has not been analyzed. For biochemical characterization of $C$. glutamicum OtsA the gene otsA was cloned into the vector pASK-IBA3+. Thereby a gene fusion was generated, which encodes a C-terminal Strep-tagged OtsA variant for purification via affinity chromatography. For in vitro characterization of the substrate specificity of the $53 \mathrm{kDa}$ OtsA-Strep construct, the protein was purified via StrepTactin affinity chromatography (Additional file 1: Fig. S1). Specific activities of $0.29 \pm 0.01 \mu \mathrm{mol} \mathrm{min}{ }^{-1}$ (mg protein $)^{-1}$ and $0.15 \pm 0.01$ were determined when $5 \mathrm{mM}$ of UDP-glucose and ADP-glucose, respectively, were used as substrate for purified OtsA-Strep. Deletion of otsA improved $\alpha$ GG production of the strain C. glutamicum $\triangle$ otsA (pEKEx3-ggpSP) when compared to C. glutamicum (pEKEx3-ggpSP) (Table 3). This indicates that supply of the precursor ADP-glucose was improved by deletion of otsA in C. glutamicum. Taken together it can be concluded that C. glutamicum OtsA used UDP- as well as ADP-glucose as precursor for trehalose-phosphate synthesis and therefore ots $A$ is an important target for the metabolic engineering towards improved $\alpha \mathrm{GG}$ synthesis.

In a next step $g \lg A$ was inactivated by insertion mutagenesis in C. glutamicum $\Delta$ otsA. Upon plasmid encoded overexpression of ggpS and ggpP the resulting strain C. glutamicum DotsA IMglgA (pEKEx3-ggpSP) formed during cultivation in minimal medium with $2 \%$ (w/v) sucrose and $750 \mathrm{mM} \mathrm{NaCl} 4.22 \pm 0.22 \mathrm{mM} \alpha \mathrm{GG}$, which is more than the amounts of $\alpha \mathrm{GG}$ formed by each of the parental strains (Table 3). As this increased product formation by C. glutamicum $\triangle$ otsA IMglgA (pEKEx3ggpSP) is achieved by the use of nearly the same amount of substrate, the yield $(\mathrm{p} / \mathrm{s})$ is further increased when compared to the predecessor strains (Table 3).

The properties of the strain C. glutamicum $\triangle$ otsA IMglgA (pEKEx3-ggpSP) were also tested in batch-fermentations in small, $\mathrm{pH}$-controlled, aerated $1 \mathrm{~L}$ bioreactors in $\mathrm{CgC}$-minimal medium with $2 \%(\mathrm{w} / \mathrm{v})$ sucrose as sole source of carbon and energy. As depicted in Fig. 3, growth of C. glutamicum $\Delta$ otsA IMglgA (pEKEx3ggpSP) slowed down and production of $\alpha \mathrm{GG}$ set in after $4 \mathrm{~h}$ of cultivation, when the $\mathrm{NaCl}$ (final concentration $750 \mathrm{mM}$ ) was added to the culture. Production of $\alpha \mathrm{GG}$, slow growth and concomitant consumption of sucrose continued until a concentration of about $3.4 \pm 0.5 \mathrm{mM}$

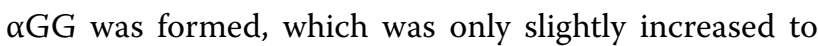
$3.9 \pm 0.5 \mathrm{mM}$ upon prolonged incubation. After about $20 \mathrm{~h}$ of cultivation both growth and $\alpha \mathrm{GG}$ production ceased nearly completely albeit a concentration of about $35.4 \pm 3.4 \mathrm{mM}$ of the substrate sucrose was still present in the culture broth.

Taken together these results show, that upon heterologous expression of the genes ggpS and ggpP for aGG synthesis from Synechocystis sp. PCC 6803 C. glutamicum produces and secretes the compatible solute $\alpha \mathrm{GG}$ in response to $\mathrm{NaCl}$-induced hyperosmotic stress.

Table 3 Production of a-GG with different C. glutamicum strains carrying the plasmid pEKEx3-ggpPS during cultivation in $\mathrm{CgC}$ minimal medium with $1 \%(\mathrm{w} / \mathrm{v})$ sucrose as sole substrate

\begin{tabular}{|c|c|c|c|c|}
\hline Strain & aGG production (mM) & Biomass ( $\mathrm{g} \mathrm{cdm} \mathrm{L}^{-1}$ ) & $\begin{array}{l}\text { Substrate } \\
\text { consumption (mM) }\end{array}$ & $\begin{array}{l}\text { Yield P/S } \\
(\mathrm{mmol} \text { mol } \\
\left.\mathrm{I}^{-1}\right)\end{array}$ \\
\hline C. glutamicum (pEKEx3-ggpSP) & $0.57 \pm 0.06$ & $7.60 \pm 0.03$ & $60.82 \pm 1.59$ & $9.4 \pm 1.1$ \\
\hline C. glutamicum IMglgA (pEKEx3-ggpSP) & $3.23 \pm 0.15$ & $5.31 \pm 0.31$ & $62.23 \pm 2.63$ & $52.0 \pm 2.4$ \\
\hline C. glutamicum $\triangle$ otsA (pEKEx3-ggpSP) & $2.19 \pm 0.20$ & $4.91 \pm 0.19$ & $64.78 \pm 2.74$ & $33.8 \pm 3.1$ \\
\hline C. glutamicum IMglgA $\triangle$ otsA (pEKEx3-ggpSP) & $4.22 \pm 0.22$ & $4.31 \pm 0.23$ & $61.19 \pm 3.98$ & $69.0 \pm 3.6$ \\
\hline
\end{tabular}

Strains were inoculated at an $\mathrm{OD}_{600}$ of 1, a-GG synthesis was induced by addition of $\mathrm{NaCl}$ (final concentration $750 \mathrm{mM}$ ) after $4 \mathrm{~h}$ of cultivation. The fermentations were performed in triplicate, samples of each fermentation were analyzed in triplicate, means are from the three independent experiments; the indicated experimental error means standard deviation 


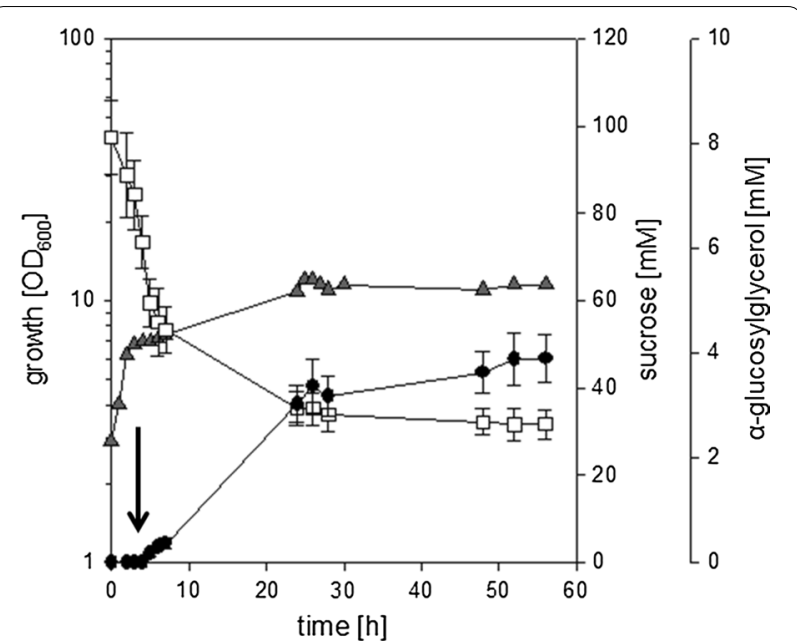

Fig. 3 aGG accumulation during cultivation of C. glutamicum IMglgA $\triangle o t s A$ (pEKEx3-ggpSP) in small-scale bioreactors in CgC minimal medium with $2 \%$ sucrose. The culture was inoculated at an $\mathrm{OD}_{600}$ of about 1 , after $4 \mathrm{~h}$ of cultivation a hyperosmotic shock was applied by addition of $\mathrm{NaCl}$ (final concentration $750 \mathrm{mM}$; the timepoint of $\mathrm{NaCl}$ addition is indicated by a black arrow). Growth (grey triangles), sucrose concentration (white squares), and a-GG concentration (black circles) were analyzed throughout cultivation. The fermentation was performed in duplicates, for substrate and product concentrations samples from each fermentation were analyzed in triplicates. Means of substrate and product concentrations are from two independent experiments; error bars indicate standard deviations. For growth a representative curve of the two experiments is shown

Moreover, genetic modifications of C. glutamicum strains for an improved supply of the precursor ADP-glucose by inactivation of $g l g A$ and $o t s A$ led to an increased $\alpha \mathrm{GG}$ production.

\section{Optimization of nitrogen-supply for enhanced aGG production by $C$. glutamicum $\triangle$ otsA IMglgA (pEKEx3-ggpSP)}

The production of $\alpha \mathrm{GG}$ by C. glutamicum (pEKEX3ggpSP) correlated well with the amount of externally present $\mathrm{NaCl}$ (Fig. 2). This observation fits well to the proposed mechanism for GgpS activation by increased intracellular of sodium ions [20]. Accumulation of compatible solutes is the common way in bacteria to respond to increased sodium concentrations, which in C. glutamicum proceeds in absence of external compatible solutes via synthesis of, e.g. proline and trehalose [37]. Synthesis of trehalose is abolished in OtsA and GlgA deficient $C$. glutamicum strains such as C. glutamicum $\Delta$ otsA IMglgA [36], however depending of the availability of suitable nitrogen-sources proline is accumulated as main compatible solute [37]. As intracellular proline accumulation might counteract the conditions required for efficient $\alpha G G$ synthesis, effects of limited nitrogen availability on $\alpha \mathrm{GG}$ production with C. glutamicum strains were tested. To optimize nitrogen supply for an optimal GG production in the presence of still sufficient growth, $C$. glutamicum (pEKEx3-ggpSP) was cultivated in CgCminimal medium without ammonium sulfate and varying amounts of urea $\left(0 ; 0.1,0.5,1.0,5 \mathrm{~g} \mathrm{~L}^{-1}\right) . \mathrm{NaCl}$ (final concentration $750 \mathrm{mM}$ ) was added to the cultures after $4 \mathrm{~h}$ of cultivation to induce $\alpha \mathrm{GG}$ production. Neither growth nor $\alpha$ GG production were observed for cultivations in the medium without urea (Fig. 4a). In medium containing $5 \mathrm{~g} \mathrm{~L}^{-1}$ urea growth (Fig. 4a), sucrose utilization (Fig. 4b), and $\alpha \mathrm{GG}$ production (Fig. 4c) proceeded as observed for $\mathrm{CgC}$-minimal medium. Also for the media containing $0.1,0.5$, and $1.0 \mathrm{~g} \mathrm{~L}^{-1}$ urea growth of C. glutamicum (pEKEx3-ggpSP) was observed. The final ODs increased in parallel to the increase of urea in the medium, but the final ODs of these cultures remained significantly below the final ODs observed for the cultivations with $5.0 \mathrm{~g} \mathrm{~L}^{-1}$ urea and in $\mathrm{CgC}$ minimal medium, which contains $5 \mathrm{~g} \mathrm{~L}^{-1}$ urea plus $5 \mathrm{~g} \mathrm{~L}^{-1}$ ammonia chloride as nitrogen sources (Fig. 4a). This result indicates that different levels of N-limitation were installed in these cultivations. Concerning the production of $\alpha \mathrm{GG}$ in N-limited cultures, the highest amount of $2.7 \mathrm{mM}$ was produced in cultivations of C. glutamicum (pEKEx3-ggpSP) with $1.0 \mathrm{~g} \mathrm{~L}^{-1}$ urea (Fig. 4c); however, the initially provided sucrose was not completely consumed after $24 \mathrm{~h}$ of cultivation (Fig. 4b).

Effects of nitrogen limitation on the $\alpha G G$ production were also tested for the strains with optimized provision of the precursor ADP-glucose. As depicted in Table 4 $\alpha G G$ production after the hyperosmotic shock by $\mathrm{NaCl}$ addition was significantly increased for all strains tested, e.g. C. glutamicum $\Delta$ otsA IMglgA (pEKEx3-ggpSP) produced $6.40 \pm 0.88 \mathrm{mM} \alpha \mathrm{GG}$ in the cultivations with $1.0 \mathrm{~g} \mathrm{~L}^{-1}$ urea, which is nearly twice the amount of the $\alpha G G$ produced in cultivations in $\mathrm{CgC}$ minimal medium (Table 3). As all tested strains consumed in nitrogen limited cultivations only about half of the amount of sucrose they consumed in non-limited cultivations, the $\alpha G G$ yield (product/substrate) increased significantly (Tables 3, 4).

The effects of N-limitation on $\alpha \mathrm{GG}$ production with the strain C. glutamicum DotsA IMglgA (pEKEx3ggpSP) were also tested in batch-fermentations in a small, $\mathrm{pH}$-controlled, aerated bioreactor in a 1-L scale in minimal medium with $1.0 \mathrm{~g} \mathrm{~L}^{-1}$ urea as sole nitrogen source and $2 \%(\mathrm{w} / \mathrm{v})$ sucrose as sole source of carbon and energy. As described above for conditions of sufficient nitrogen supply, growth of $C$. glutamicum $\triangle$ otsA IMglgA (pEKEx3-ggpSP) slowed down and production of $\alpha \mathrm{GG}$ set in after $4 \mathrm{~h}$ of cultivation, when $\mathrm{NaCl}$ (final concentration $750 \mathrm{mM}$ ) was added to the culture (Fig. 5). In difference to the cultivation in $\mathrm{CgC}$ 

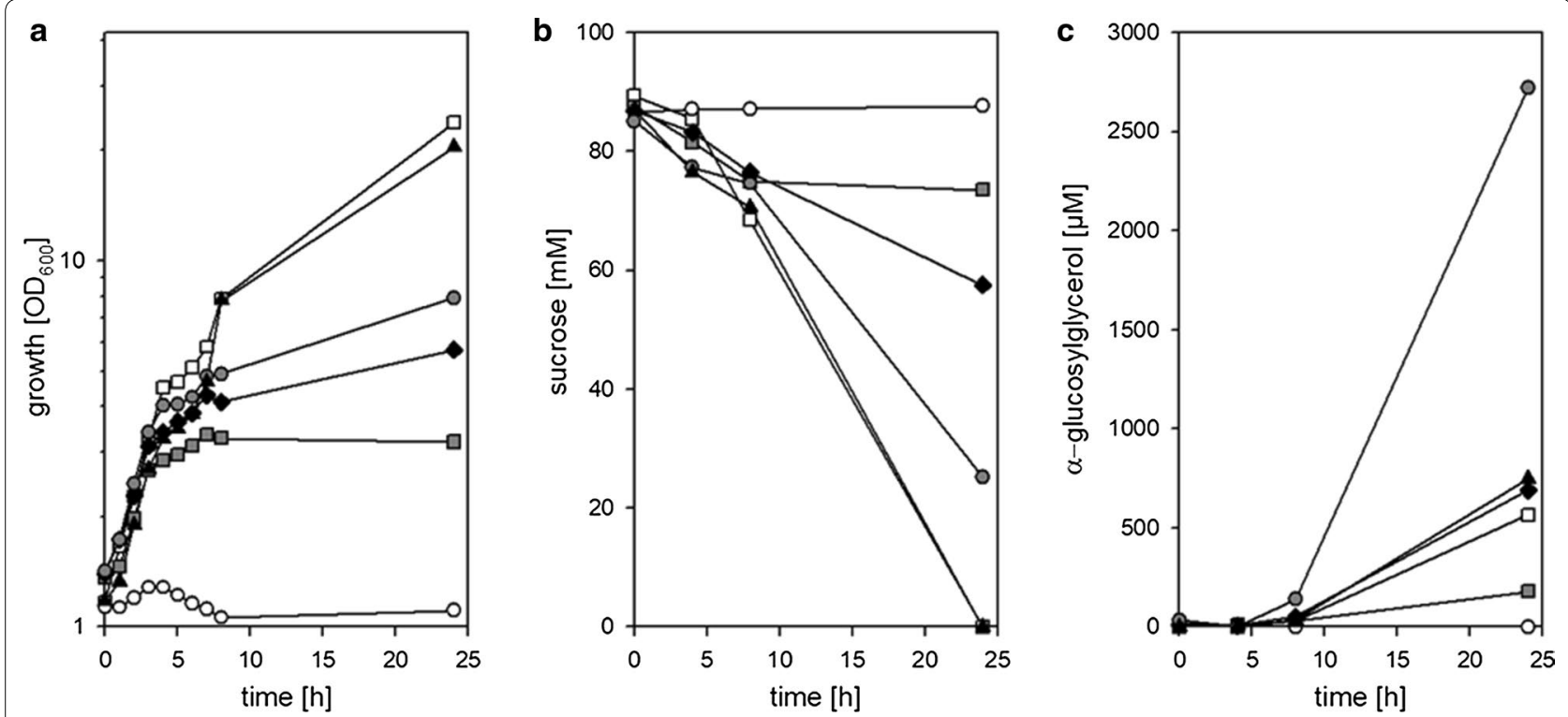

Fig. 4 Effect of nitrogen limitation on a-GG production with C. glutamicum (pEKEx3-ggpSP). Growth a, substrate consumption b and aGG accumulation c were determined for C. glutamicum (pEKEx3-ggpSP) in the course of cultivation in CgC-minimal medium (open squares), or CgC-minimal medium without ammonia and $0 \mathrm{~g} \mathrm{~L}^{-1}$ urea (white circles), $0.1 \mathrm{~g} \mathrm{~L}^{-1}$ urea (grey squares), $0.5 \mathrm{~g} \mathrm{~L}^{-1}$ urea (solid diamonds), $1.0 \mathrm{~g} \mathrm{~L}{ }^{-1}$ urea (grey circles), or $5.0 \mathrm{~g} \mathrm{~L}^{-1}$ urea (solid triangles). Data from one representative experiment of a series of three are shown

Table 4 Production of a-GG with different C. glutamicum strains carrying the plasmid pEKEx3-ggpPS during cultivation minimal medium with $1 \%(\mathrm{w} / \mathrm{v})$ sucrose as sole substrate and $1.0 \mathrm{~g} \mathrm{~L}^{-1}$ urea as sole nitrogen source

\begin{tabular}{|c|c|c|c|c|}
\hline Strain & aGG production (mM) & Biomass ( $\mathrm{g} \mathrm{cdm} \mathrm{L}^{-1}$ ) & $\begin{array}{l}\text { Substrate } \\
\text { consumption } \\
\text { (mM) }\end{array}$ & Yield $\mathrm{P} / \mathrm{S}\left(\mathrm{mmol} \mathrm{mol}^{-1}\right)$ \\
\hline C. glutamicum (pEKEx3-ggpSP) & $2.69 \pm 0.46$ & $2.34 \pm 0.06$ & $38.04 \pm 0.98$ & $70.9 \pm 14.0$ \\
\hline C. glutamicum IMglgA (pEKEx3-ggpSP) & $5.24 \pm 0.57$ & $1.80 \pm 0.01$ & $35.70 \pm 5.90$ & $146.6 \pm 16.1$ \\
\hline C. glutamicum $\triangle$ otsA (pEKEx3-ggpSP) & $5.54 \pm 0.60$ & $2.39 \pm 0.03$ & $35.24 \pm 3.13$ & $157.4 \pm 17.0$ \\
\hline C. glutamicum IMglgA $\triangle$ otsA (pEKEX3-ggpSP) & $6.40 \pm 0.88$ & $2.20 \pm 0.01$ & $34.41 \pm 4.36$ & $186.1 \pm 25.5$ \\
\hline
\end{tabular}

Strains were inoculated at an $\mathrm{OD}_{600}$ of 1 , a-GG synthesis was induced by addition of $\mathrm{NaCl}$ (final concentration $750 \mathrm{mM}$ ) after $4 \mathrm{~h}$ of cultivation. The fermentations were performed in triplicate, samples of each fermentation were analyzed in triplicate, means are from the three independent experiments; the indicated experimental error means standard deviation

minimal medium after prolonged incubation the biomass in the culture liquid significantly decreased. This decrease coincided with the formation of foam and especially cell aggregates in the bioreactor, which occurred even though anti-foam reagent was present in the cultivation broth. Biomass was found sticking to all components (e.g. stirrer, gas inlet, and electrodes) and the glass wall in the headspace of the fermenter. Despite the loss of biomass in the culture liquid production of $\alpha \mathrm{GG}$ and concomitant sucrose consumption slowly continued until the cultivation was ended $58 \mathrm{~h}$ after the inoculation, when a final concentration of $8.4 \pm 0.9 \mathrm{mM} \alpha \mathrm{GG}$ was present in the culture liquid. In the course of the fermentation $48.2 \pm 8.1 \mathrm{mM}$ sucrose have been consumed in total, which results in a yield of about $0.17 \mathrm{~mol} \mathrm{~mol}^{-1}$. These results show that by combining strain development with optimized cultivation conditions such as nitrogen limitation efficient $\alpha \mathrm{GG}$ production with recombinant $C$. glutamicum strains was possible. However, the high amounts of residual sucrose observed here during cultivations of C. glutamicum $\triangle$ otsA IMglgA (pEKEx3-ggpSP) exclusively in stirred bioreactors (Figs. 3,5 ) and especially the strong formation of foam and cell aggregates observed for $\mathrm{N}$-limited cultures indicate, that mechanical robustness of the cells might be compromised by the here applied strategies to limit synthesis of compatible solutes. 


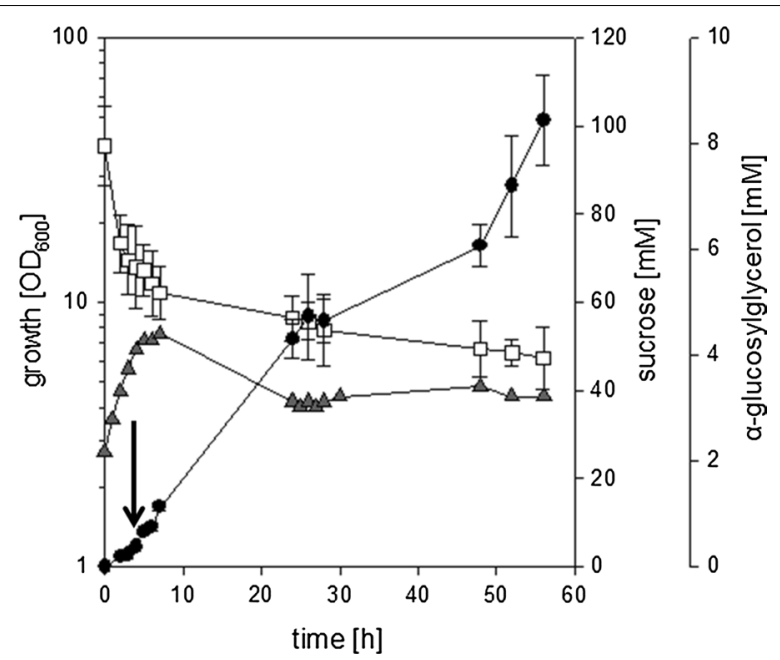

Fig. 5 Effect of nitrogen limitation on aGG accumulation during cultivation of C. glutamicum IMglgA $\triangle$ otsA (pEKEx3-ggpSP) in small-scale bioreactors in $\mathrm{CgC}$ minimal medium with $2 \%$ sucrose. The culture was inoculated at an $\mathrm{OD}_{600}$ of about 1, after $4 \mathrm{~h}$ of cultivation a hyperosmotic shock was applied by addition of $\mathrm{NaCl}$ (final concentration $750 \mathrm{mM}$; the timepoint of $\mathrm{NaCl}$ addition is indicated by a black arrow). Growth (grey triangles), sucrose concentration (open squares), and a-GG concentration (solid circles) were analyzed throughout cultivation. The fermentation was performed in duplicates, for substrate and product concentrations samples from each fermentation were analyzed in triplicates. Means of substrate and product concentrations are from two independent experiments; error bars indicate standard deviations. For growth a representative curve of the two experiments is shown

\section{Analysis of aGG production in the trehalose synthesis deficent strain C. glutamicum $\Delta$ ots $A \Delta$ treY $\Delta$ treS (pEKEx3-ggpSP)}

Beside its role as a compatible solute trehalose is in $C$. glutamicum a precursor for the synthesis of trehalose mycolates [36, 37], which are important building blocks of the mycolic acid layer, a second permeability barrier functionally similar to the Gram-negative outer membrane [54, 55]. For the trehalose synthesis and thus mycolic acid layer deficient strain C. glutamicum
$\Delta$ ots $A \Delta$ tre $Y \Delta$ treS besides increased osmo-sensitivity and aggregation of cells in liquid culture was reported [56]. Inactivation of $g l g A$ impairs synthesis of glycogen, which acts as precursor for trehalose synthesis via the TreYZ pathway [36]. Thus redirection of the carbon flux in $C$. glutamicum $\triangle$ otsA IMglgA (pEKEx3-ggpSP) probably resulted in a lack of trehalose, which is required for the synthesis of the mycolic acid layer. The increased sensitivity to osmotic stress and tendency to form cell aggregates might underlie the in-complete substrate utilization and loss of biomass observed during cultivation in stirred bioreactors. To rule out that trehalose-synthesis deficiency is the reason for the improved $\alpha G G$ production of C. glutamicum $\triangle$ otsA IMglgA (pEKEx3-ggpSP) when compared to C. glutamicum IMglgA (pEKEx3-ggpSP) or C. glutamicum $\Delta$ otsA (pEKEx3-ggpSP) (Tables 3, 4), aGG production of C. glutamicum $\Delta$ ots $A \Delta$ treY $\triangle$ treS (pEKEx3-ggpSP) was analyzed. Accumulation of $\alpha \mathrm{GG}$ in the culture broth and $\alpha \mathrm{GG}$ productivity of C. glutamicum $\Delta$ otsA $\Delta$ treY $\Delta$ treS (pEKEx3-ggpSP) in cultivations in $\mathrm{CgC}$-minimal medium as well as $\mathrm{N}$-limited medium were significantly lower (Table 5) than the parameters determined for C. glutamicum $\triangle$ otsA IMglgA (pEKEx3ggpSP) and quite similar to the parameters determined for C. glutamicum $\Delta$ otsA (pEKEx3-ggpSP) (Tables 3, 4), which is still able to synthesize trehalose [37]. Taken together these results show that indeed the strategy to increase availability of the precursor ADP-glucose by inactivation of $g \lg A$ and deletion of $o t s A$ was responsible for the improved $\alpha \mathrm{GG}$ by $C$. glutamicum $\triangle$ otsA IMglgA (pEKEx3-ggpSP) and not the concomitantly obtained trehalose-synthesis deficiency.

\section{Discussion}

The here developed microbial production of the compatible solute $\alpha \mathrm{GG}$ using a genetically engineered C. glutamicum strain offers an alternative to the hitherto described enzymatic syntheses of this compound [15] and $\alpha \mathrm{GG}$ production with an genetically engineered strain of Synechocystis sp. PCC 6803 [26]. Microbial synthesis of $\alpha \mathrm{GG}$

Table 5 Production of a-GG with the trehalose synthesis deficient strain C. glutamicum $\triangle$ otsA $\triangle$ treY $\triangle$ treS (pEKEx3-ggpPS) during cultivation with $1 \%(\mathrm{w} / \mathrm{v})$ sucrose as sole substrate in $\mathrm{CgC}$ minimal medium or minimal medium and $1.0 \mathrm{~g} \mathrm{L^{-1 }}$ urea as sole nitrogen source

\begin{tabular}{|c|c|c|c|c|}
\hline Medium & aGG production (mM) & Biomass ( $\mathrm{g} \mathrm{cdm} \mathrm{L}^{-1}$ ) & $\begin{array}{l}\text { Substrate } \\
\text { consumption (mM) }\end{array}$ & Yield P/S (mmol mol l-1) \\
\hline CgC-minimal medium & $2.83 \pm 0.37$ & $4.33 \pm 0.41$ & $63.67 \pm 2.86$ & $44.40 \pm 5.76$ \\
\hline $\begin{array}{l}\text { Minimal medium with } \\
\qquad 1.0 \mathrm{~g} \mathrm{~L}^{-1} \text { urea }\end{array}$ & $3.91 \pm 0.48$ & $1.85 \pm 0.06$ & $31.48 \pm 1.36$ & $124.23 \pm 15.40$ \\
\hline
\end{tabular}

Cultures were inoculated at an $\mathrm{OD}_{600}$ of $1, \mathrm{a}-\mathrm{GG}$ synthesis was induced by addition of $\mathrm{NaCl}$ (final concentration $750 \mathrm{mM}$ ) after $4 \mathrm{~h}$ of cultivation. The fermentations were performed in triplicate, samples of each fermentation were analyzed in triplicate, means are from the three independent experiments; the indicated experimental error means standard deviation 
allows a large flexibility for the use of different sources of carbon, which is not the case for the currently described processes for enzymatic $\alpha$ GG synthesis [22, 23]. The engineered Synechocystis sp. PCC 6803 strain produced about $1 \mathrm{mM}$ of extracellular $\alpha \mathrm{GG}$ during a production cycle of 6 days in a photobioreactor [26]. In this study more than 6 times higher $\alpha \mathrm{GG}$ concentrations were obtained in cultivations of C. glutamicum $\triangle$ otsA IMglgA (pEKEx3-ggpSP) during $24 \mathrm{~h}$, however sucrose was here used as source of carbon and energy. A drawback of the two microbial approaches to produce $\alpha G G$ is the need to induce $\alpha G G$ synthesis by addition of $\mathrm{NaCl}$ to the cultivation broth. Thus for further utilization of the produced $\alpha \mathrm{GG} \mathrm{NaCl}$ has to be removed in the course of the downstream processing. However, this technical problem of the current concept of $\alpha \mathrm{GG}$ production with C. glutamicum strains overexpressing ggpS and ggpP from Synechocystis sp. PCC 6803 illustrates the intrinsic mode of activity control of the GGPS. Similar to the homologous situation in Synechocystis sp. PCC 6803 [20, 26] NaCl stress was shown to be required to initiate $\alpha \mathrm{GG}$ synthesis in C. glutamicum (pEKEx3-ggpSP) cells, which contained the preformed $\alpha \mathrm{GG}$ biosynthesis enzymes GGPS and GGPP. This result indicates that the mechanism of osmosensing of GGPS by salt-dependent protein-nucleic acid interactions recently described for the first time using in vitro studies [20] is conserved even when the enzyme is transferred into a heterologous host. Elimination of the intrinsic activity control of GgpS activity would probably cancel out the need of $\mathrm{NaCl}$ addition to the culture broth, which is currently required to start $\alpha$ GG synthesis. This approach should enable $\alpha \mathrm{GG}$ production under normal osmotic conditions, however, the molecular basis underlying inhibition of GgpS activity by binding to DNA has not been elucidated so far.

In this context, the observed export of $\alpha \mathrm{GG}$ is interesting in terms of mechanistic aspects. Under the applied experimental conditions, C. glutamicum secretes this compound into the surrounding medium, which raises at least two different questions, in view of the fact that $\alpha G G$, although being a well-known compatible solute, is a foreign compound in the heterologous host, C. glutamicum: (i) By which kind of mechanism is $\alpha \mathrm{GG}$ exported out of the C. glutamicum cell, and (ii) why is aGG continuously secreted albeit C. glutamicum could use it as compatible solute? In case of hypo-osmotic conditions, compatible solutes are in general expelled out of the cell via so-called mechanosensitive channels, and C. glutamicum possesses at least two of them, an MscS-type and an MscL-type channel $[57,58]$. On first glance, it seems very unlikely that $\alpha \mathrm{GG}$ uses this kind of pathway to leave the cell, since export of this compound was observed under hyperosmotic stress and mechanosensitive channels are closed under these conditions [59]. Consequently, it seems likely that $\alpha G G$ is secreted via a so far unknown solute export mechanism, most probably a general transport system devoted to the export of unwanted compounds out of the cytoplasm. The intracellular accumulation of $450 \mathrm{mM} \alpha \mathrm{GG}$ indicates that export does not proceed very efficiently, which is not surprising as $\alpha \mathrm{GG}$ is for C. glutamicum a foreign molecule neither synthesized nor consumed by this bacterium. On a second glance the high intracellular $\alpha G G$ concentration might allow also export of $\alpha \mathrm{GG}$ via the mechanosensitive channels despite the hyperosmotic growth conditions. In C. glutamicum the mechanosensitive channel MscCG contributes under hyperosmotic stress conditions to the fine-tuning of the concentration of compatible solutes in the cytoplasm, which are accumulated, e.g. via the betaine transporter BetP [58]. According to the "pump and leak" model for the control of the steady state level of compatible solutes in the cytoplasm export of compatible solutes via MscCG compensates for their unbalanced uptake [58, 60]. In a similar manner the MscCG might export $\alpha \mathrm{GG}$ when its intracellular accumulation requires to be compensated despite cultivation in presence of high $\mathrm{NaCl}$ concentrations.

The second question raised above is even more surprising. Considering the fact that C. glutamicum (pEKEx2ggpSP) has to counteract hyperosmotic stress in the presence of an external concentration of $750 \mathrm{mM} \mathrm{NaCl}$ and is capable by synthesis of its genuine compatible solutes, e.g. proline or trehalose, to counteract this stress it seems surprising that $\alpha$ GG synthesis does not cease in the course of cultivation. In Synechocystis sp. PCC 6803 the physical stimulus for the synthesis of the compatible solute $\alpha \mathrm{GG}$ is not osmotic stress: Synthesis of $\alpha \mathrm{GG}$ exclusively proceeds in Synechocystis sp. PCC 6803 in response to ionic stress caused by addition of salts such as $\mathrm{NaCl}$ and not in response to addition of compounds like sucrose, which only increases the osmolality of the medium [46]. Different from the situation in Synechocystis sp. PCC 6803 addition of ionic compounds like $\mathrm{NaCl}$ as well as non-ionic compounds like sorbitol to the culture broth activate in C. glutamicum the hyperosmotic stress response, which in this bacterium comprises synthesis and/or uptake of compatible solutes [35] but also the uptake of potassium ions as an immediate reaction [61]. Taken together, the differences in the osmo- and ionic stress responses in Synechocystis sp. PCC 6803 and C. glutamicum might account for the continuous synthesis of $\alpha \mathrm{GG}$ in C. glutamicum (pEKEx2-ggpSP) upon the addition of $\mathrm{NaCl}$ to the medium.

The export of $\alpha \mathrm{GG}$ observed for the C. glutamicum strains overexpressing ggpSP allows an indirect insight into the capacity of $C$. glutamicum for glycogen 
synthesis. C. glutamicum transiently accumulates large amounts of glycogen when cultivated in presence of sugars [41], however the internal glycogen amount always depends on the rates for synthesis and degradation $[38,39]$. Therefore $\alpha$ GG synthesis by GGPS and GGPP depends on the availability of the precursors glycerol 3-phosphate and ADP-glucose, which is the central intermediate of glycogen synthesis [62]. Availability of ADP-glucose dictates the rate of glycogen synthesis in bacteria as the rate of its synthesis by the ADP-glucose pyrophosphorylase is controlled in bacteria by multiple mechanisms $[63,64]$. Redirection of the flux from glycogen synthesis to $\alpha G G$ synthesis by inactivation of $g \lg A$ allows to assess a rate of ADP-glucose formation and thus precursor supply for glycogen synthesis in C. glutamicum, as $\alpha \mathrm{GG}$ is constantly exported and not consumed. The continuous production of $\alpha \mathrm{GG}$ reveals a continuous provision of the precursor ADPglucose, which indicates the presence of a continuous flux towards glycogen synthesis in C. glutamicum. This consideration based on the continuously observed $\alpha \mathrm{GG}$ accumulation fits within the proposed model of glycogen as a carbon capacitor in bacteria [38, 65-67]. The proportion of flux towards glycogen might be even slightly higher as here indicated by the synthesis of provision of $\alpha \mathrm{GG}$, as availability of the precursor glycerol 3-phosphate might here be limiting $\alpha$ GG synthesis. Taken together, the constant accumulation of $\alpha \mathrm{GG}$ in the supernatant of C. glutamicum $\Delta$ otsA IMglgA (pEKEx3-ggpSP) indicates that a high proportion of the glycolytic flux can be channeled into glycogen, which vice versa is a prerequisite for a continuous supply of the precursor ADP-glucose allowing efficient microbial production of $\alpha \mathrm{GG}$ in C. glutamicum.

\section{Additional file}

Additional file 1: Fig. S1. Purification of recombinant streptavidin-tagged C. glutamicum OtsA from E. coli BL21(DE3)(pASK-IBA3-otsA): SDS-PAGE analysis of crude cell extract and Streptavidin-affinity chromatography flow-through, wash fraction, and elution fractions. PAGE ruler prestained protein ladder (MBI Fermentas) was used as marker.

\section{Abbreviations}

cdm: cell dry mass; WT: wild-type; aGG: a-D-glucosylglycerol.

\section{Authors' contributions}

GMS, RK, KM and BR designed and coordinated the study. GMS, BR, NB, and SD carried out the experiments and analyzed the results. GMS and RK wrote the manuscript. All authors read and approved the final manuscript.

\section{Author details}

${ }^{1}$ Institute of Biochemistry, University of Cologne, Zülpicher Str. 47, 50674 Cologne, Germany. ${ }^{2}$ Institute of Microbiology and Biotechnology, Ulm University, Albert-Einstein Allee 11, 89081 Ulm, Germany. ${ }^{3}$ Present Address: Gutachterbüro U. Borchardt, Hennef (Sieg), Germany. ${ }^{4}$ Present Address: Evonik Degussa GmbH, Halle (Westphalia), Germany.

\section{Acknowledgements}

The authors thank Eva Glees and Ute Meyer for excellent technical assistance and bitop AG (Witten, Germany) for providing aGG.

\section{Competing interests}

The authors declare that the research was conducted in the absence of any commercial or financial relationships that could be construed as a potential competing interests.

\section{Availability of data and materials}

Not applicable.

Ethics approval and consent to participate

Not applicable.

Funding

Not applicable.

\section{Publisher's Note}

Springer Nature remains neutral with regard to jurisdictional claims in published maps and institutional affiliations.

Received: 16 January 2018 Accepted: 5 June 2018

Published online: 16 June 2018

\section{References}

1. Wood JM. Osmosensing by bacteria: signals and membrane-based sensors. Microbiol Mol Biol Rev. 1999;63:230-62.

2. da Costa MS, Santos H, Galinski EA. An overview of the role and diversity of compatible solutes in bacteria and archaea. Adv Biochem Eng Biotechnol. 1998;61:117-53.

3. Borowitzka LJ, Demmerle S, Mackay MA, Norton RS. Carbon-13 nuclear magnetic resonance study of osmoregulation in a blue-green alga. Science. 1980;210:650-1.

4. Faria TQ, Knapp S, Ladenstein R, Macanita AL, Santos H. Protein stabilisation by compatible solutes: effect of mannosylglycerate on unfolding thermodynamics and activity of ribonuclease A. ChemBioChem. 2003:4:734-41.

5. Hincha DK, Hagemann M. Stabilization of model membranes during drying by compatible solutes involved in the stress tolerance of plants and microorganisms. Biochem J. 2004;383:277-83.

6. Sawangwan T, Goedl C, Nidetzky B. Glucosylglycerol and glucosylglycerate as enzyme stabilizers. Biotechnol J. 2010;5:187-91.

7. Kempf B, Bremer E. Uptake and synthesis of compatible solutes as microbial stress responses to high-osmolality environments. Arch Microbiol. 1998;170:319-30

8. Krämer R. Bacterial stimulus perception and signal transduction: response to osmotic stress. Chem Rec. 2010;10:217-29.

9. Pastor JM, Salvador M, Argandona M, Bernal V, Reina-Bueno M, Csonka LN Iborra JL, Vargas C, Nieto JJ, Canovas M. Ectoines in cell stress protection: uses and biotechnological production. Biotechnol Adv. 2010;28:782-801.

10. Graf R, Anzali S, Buenger J, Pfluecker F, Driller H. The multifunctional role of ectoine as a natural cell protectant. Clin Dermatol. 2008;26:326-33.

11. Lentzen G, Schwarz T. Extremolytes: natural compounds from extremophiles for versatile applications. Appl Microbiol Biotechnol. 2006;72:623-34.

12. Loi P, luso D, Czernik M, Zacchini F, Ptak G. Towards storage of cells and gametes in dry form. Trends Biotechnol. 2013;31:688-95.

13. Julca I, Alaminos M, Gonzalez-Lopez J, Manzanera M. Xeroprotectants for the stabilization of biomaterials. Biotechnol Adv. 2012;30:1641-54.

14. Luley-Goedl C, Nidetzky B. Glycosides as compatible solutes: biosynthesis and applications. Nat Prod Rep. 2011;28:875-96.

15. Tan X, Luo Q, Lu X. Biosynthesis, biotechnological production, and applications of glucosylglycerols. Appl Microbiol Biotechnol. 2016;100:6131-9.

16. Klähn S, Steglich C, Hess WR, Hagemann M. Glucosylglycerate: a secondary compatible solute common to marine cyanobacteria from nitrogenpoor environments. Environ Microbiol. 2010;12:83-94. 
17. Hagemann M. Molecular biology of cyanobacterial salt acclimation. FEMS Microbiol Rev. 2011;35:87-123.

18. Marin K, Zuther E, Kerstan T, Kunert A, Hagemann M. The ggpS gene from Synechocystis sp. strain PCC 6803 encoding glucosyl-glycerol-phosphate synthase is involved in osmolyte synthesis. J Bacteriol. 1998;180:4843-9.

19. Hagemann M, Schoor A, Jeanjean R, Zuther E, Joset F. The stpA gene form Synechocystis sp. strain PCC 6803 encodes the glucosylglycerolphosphate phosphatase involved in cyanobacterial osmotic response to salt shock. J Bacteriol. 1997:179:1727-33.

20. Novak JF, Stirnberg M, Roenneke B, Marin K. A novel mechanism of osmosensing, a salt-dependent protein-nucleic acid interaction in the cyanobacterium Synechocystis species PCC 6803. J Biol Chem. 2011;286:3235-41.

21. Ojima T, Saburi W, Yamamoto T, Kudo T. Characterization of Halomonas sp. strain $\mathrm{H} 11$ alpha-glucosidase activated by monovalent cations and its application for efficient synthesis of alpha-D-glucosylglycerol. Appl Environ Microbiol. 2012;78:1836-45.

22. Jeong JW, Seo DH, Jung JH, Park JH, Baek NI, Kim MJ, Park CS. Biosynthesis of glucosyl glycerol, a compatible solute, using intermolecular transglycosylation activity of amylosucrase from Methylobacillus flagellatus KT. Appl Biochem Biotechnol. 2014;173:904-17.

23. Goedl C, Sawangwan T, Mueller M, Schwarz A, Nidetzky B. A high-yielding biocatalytic process for the production of 2-O-(alpha-D-glucopyranosyl)sn-glycerol, a natural osmolyte and useful moisturizing ingredient. Angew Chem Int Ed Engl. 2008;47:10086-9.

24. Eikmanns BJ, Blombach B. The pyruvate dehydrogenase complex of Corynebacterium glutamicum: an attractive target for metabolic engineering. J Biotechnol. 2014;192(Pt B):339-45.

25. Becker J, Wittmann C. Bio-based production of chemicals, materials and fuels-Corynebacterium glutamicum as versatile cell factory. Curr Opin Biotechnol. 2012;23:631-40

26. Tan X, Du W, Lu X. Photosynthetic and extracellular production of glucosylglycerol by genetically engineered and gel-encapsulated cyanobacteria. Appl Microbiol Biotechnol. 2015;99:2147-54.

27. Eggeling L, Bott M. A giant market and a powerful metabolism: L-lysine provided by Corynebacterium glutamicum. Appl Microbiol Biotechnol. 2015;99:3387-94.

28. Lee JY, Na YA, Kim E, Lee HS, Kim P. The actinobacterium Corynebacterium glutamicum, an industrial workhorse. J Microbiol Biotechnol. 2016;26:807-22.

29. Heider SA, Wendisch VF. Engineering microbial cell factories: metabolic engineering of Corynebacterium glutamicum with a focus on non-natural products. Biotechnol J. 2015;10:1170-84.

30. Padilla L, Morbach S, Krämer R, Agosin E. Impact of heterologous expression of Escherichia coli UDP-glucose pyrophosphorylase on trehalose and glycogen synthesis in Corynebacterium glutamicum. Appl Environ Microbiol. 2004;70:3845-54.

31. Padilla L, Krämer R, Stephanopoulos G, Agosin E. Overproduction of trehalose: heterologous expression of Escherichia coli trehalose-6-phosphate synthase and trehalose-6-phosphate phosphatase in Corynebacterium glutamicum. Appl Environ Microbiol. 2004;70:370-6.

32. Carpinelli J, Krämer R, Agosin E. Metabolic engineering of Corynebacterium glutamicum for trehalose overproduction: role of the TreYZ trehalose biosynthetic pathway. Appl Environ Microbiol. 2006;72:1949-55.

33. Becker J, Schäfer R, Kohlstedt M, Harder BJ, Borchert NS, Stoveken N, Bremer E, Wittmann C. Systems metabolic engineering of Corynebacterium glutamicum for production of the chemical chaperone ectoine. Microb Cell Fact. 2013;12:110.

34. Pérez-García F, Ziert C, Risse JM, Wendisch VF. Improved fermentative production of the compatible solute ectoine by Corynebacterium glutamicum from glucose and alternative carbon sources. J Biotechnol. 2017:258:59-68.

35. Krämer R. Osmosensing and osmosignaling in Corynebacterium glutamicum. Amino Acids. 2009:37:487-97.

36. Tzvetkov M, Klopprogge C, Zelder O, Liebl W. Genetic dissection of trehalose biosynthesis in Corynebacterium glutamicum: inactivation of trehalose production leads to impaired growth and an altered cell wall lipid composition. Microbiology. 2003;149:1659-73.

37. Wolf A, Krämer R, Morbach S. Three pathways for trehalose metabolism in Corynebacterium glutamicum ATCC13032 and their significance in response to osmotic stress. Mol Microbiol. 2003;49:1119-34.
38. Seibold GM, Eikmanns BJ. Inactivation of the phosphoglucomutase gene pgm in Corynebacterium glutamicum affects cell shape and glycogen metabolism. Biosci Rep. 2013;33:e00059.

39. Seibold GM, Eikmanns BJ. The glgX gene product of Corynebacterium glutamicum is required for glycogen degradation and for fast adaptation to hyperosmotic stress. Microbiology. 2007;153:2212-20.

40. Clermont L, Macha A, Müller LM, Derya SM, von Zaluskowski P, Eck A, Eikmanns BJ, Seibold GM. The alpha-glucan phosphorylase MalP of Corynebacterium glutamicum is subject to transcriptional regulation and competitive inhibition by ADP-glucose. J Bacteriol. 2015;197:1394-407.

41. Seibold G, Dempf S, Schreiner J, Eikmanns BJ. Glycogen formation in Corynebacterium glutamicum and role of ADP-glucose pyrophosphorylase. Microbiology. 2007;153:1275-85.

42. Seibold GM, Breitinger KJ, Kempkes R, Both L, Krämer M, Dempf S, Eikmanns $B J$. The glgB-encoded glycogen branching enzyme is essential for glycogen accumulation in Corynebacterium glutamicum. Microbiology. 2011;157:3243-51.

43. Lindner SN, Meiswinkel TM, Panhorst M, Youn JW, Wiefel L, Wendisch VF. Glycerol-3-phosphatase of Corynebacterium glutamicum. J Biotechnol. 2012:159:216-24.

44. Sambrook J, Russel DW, Irwin N, Janssen UA. Molecular Cloning: a laboratory manual. 3rd ed. Cold Spring Harbor: Cold Spring Harbor Laboratory Press; 2001

45. Eikmanns BJ, Metzger M, Reinscheid D, Kircher M, Sahm H. Amplification of three threonine biosynthesis genes in Corynebacterium glutamicum and its influence on carbon flux in different strains. Appl Microbiol Biotechnol. 1991;34:617-22.

46. Marin K, Stirnberg M, Eisenhut M, Krämer R, Hagemann M. Osmotic stress in Synechocystis sp. PCC 6803: low tolerance towards nonionic osmotic stress results from lacking activation of glucosylglycerol accumulation. Microbiology. 2006;152:2023-30.

47. Tauch A, Kirchner O, Loffler B, Gotker S, Pühler A, Kalinowski J. Efficient electrotransformation of Corynebacterium diphtheriae with a mini-replicon derived from the Corynebacterium glutamicum plasmid pGA1. Curr Microbiol. 2002:45:362-7.

48. Dower WJ, Miller JF, Ragsdale CW. High efficiency transformation of Escherichia coli by high voltage electroporation. Nucleic Acids Res. 1988:16:6127-45.

49. Laemmli UK. Cleavage of structural proteins during the assembly of the head of bacteriophage T4. Nature. 1970;227:680-5.

50. Hagemann M, Erdmann N. Activation and pathway of glucosylglycerol synthesis in the cyanobacterium Synechocystis sp. PCC 6803. Microbiology. 1994;140:1427-31.

51. Seibold GM, Hagmann CT, Schietzel M, Emer D, Auchter M, Schreiner J, Eikmanns BJ. The transcriptional regulators RamA and RamB are involved in the regulation of glycogen synthesis in Corynebacterium glutamicum. Microbiology. 2010;156:1256-63.

52. Ruhal R, Kataria R, Choudhury B. Trends in bacterial trehalose metabolism and significant nodes of metabolic pathway in the direction of trehalose accumulation. Microb Biotechnol. 2013;6:493-502.

53. Cardoso FS, Castro RF, Borges N, Santos H. Biochemical and genetic characterization of the pathways for trehalose metabolism in Propionibacterium freudenreichii, and their role in stress response. Microbiology. 2007;153:270-80

54. Bansal-Mutalik R, Nikaido H. Quantitative lipid composition of cell envelopes of Corynebacterium glutamicum elucidated through reverse micelle extraction. Proc Natl Acad Sci USA. 2011;108:15360-5.

55. Marchand CH, Salmeron C, Bou Raad R, Meniche X, Chami M, Masi M, Blanot D, Daffe M, Tropis M, Huc E, et al. Biochemical disclosure of the mycolate outer membrane of Corynebacterium glutamicum. J Bacteriol. 2012;194:587-97.

56. Tropis M, Meniche X, Wolf A, Gebhardt H, Strelkov S, Chami M, Schomburg D, Krämer R, Morbach S, Daffe M. The crucial role of trehalose and structurally related oligosaccharides in the biosynthesis and transfer of mycolic acids in Corynebacterineae. J Biol Chem. 2005;280:26573-85.

57. Nottebrock D, Meyer U, Krämer R, Morbach S. Molecular and biochemical characterization of mechanosensitive channels in Corynebacterium glutamicum. FEMS Microbiol Lett. 2003;218:305-9.

58. Börngen K, Battle AR, Möker N, Morbach S, Marin K, Martinac B, Krämer R. The properties and contribution of the Corynebacterium glutamicum 
MscS variant to fine-tuning of osmotic adaptation. Biochim Biophys Acta. 2010;1798:2141-9.

59. Martinac B. Mechanosensitive channels in prokaryotes. Cell Physiol Biochem. 2001;11:61-76.

60. Botzenhardt J, Morbach S, Krämer R. Activity regulation of the betaine transporter BetP of Corynebacterium glutamicum in response to osmotic compensation. Biochim Biophys Acta. 2004;1667:229-40.

61. Follmann M, Becker M, Ochrombel I, Ott V, Krämer R, Marin K. Potassium transport in Corynebacterium glutamicum is facilitated by the putative channel protein $\mathrm{CgIK}$, which is essential for $\mathrm{pH}$ homeostasis and growth at acidic pH. J Bacteriol. 2009;191:2944-52.

62. Wilson WA, Roach PJ, Montero M, Baroja-Fernandez E, Munoz FJ, Eydallin G, Viale AM, Pozueta-Romero J. Regulation of glycogen metabolism in yeast and bacteria. FEMS Microbiol Rev. 2010;34:952-85.

63. Preiss J. Bacterial glycogen synthesis and its regulation. Annu Rev Microbiol. 1984;38:419-58.

64. Ballicora MA, Iglesias AA, Preiss J. ADP-glucose pyrophosphorylase, a regulatory enzyme for bacterial glycogen synthesis. Microbiol Mol Biol Rev. 2003;67:213-25.

65. Goh YJ, Klaenhammer TR. Insights into glycogen metabolism in Lactobacillus acidophilus: impact on carbohydrate metabolism, stress tolerance and gut retention. Microb Cell Fact. 2014;13:94.
66. Dauvillee D, Kinderf IS, Li Z, Kosar-Hashemi B, Samuel MS, Rampling L, Ball $\mathrm{S}$, Morell MK. Role of the Escherichia coliglgX gene in glycogen metabolism. J Bacteriol. 2005;187:1465-73.

67. Belanger AE, Hatfull GF. Exponential-phase glycogen recycling is essential for growth of Mycobacterium smegmatis. J Bacteriol. 1999;181:6670-8.

68. Hanahan D. Studies on transformation of Escherichia coli with plasmids. J Mol Biol. 1983;166:557-80.

69. Studier FW, Moffatt BA. Use of bacteriophage T7 RNA polymerase to direct selective high-level expression of cloned genes. J Mol Biol. 1986;189:113-30.

70. Eikmanns BJ, Kleinertz E, Liebl W, Sahm H. A family of Corynebacterium glutamicum/Escherichia coli shuttle vectors for cloning, controlled gene expression, and promoter probing. Gene. 1991;102:93-8.

71. Stansen C, Uy D, Delaunay S, Eggeling L, Goergen JL, Wendisch VF. Characterization of a Corynebacterium glutamicum lactate utilization operon induced during temperature-triggered glutamate production. Appl Environ Microbiol. 2005;71:5920-8.
Ready to submit your research? Choose BMC and benefit from:

- fast, convenient online submission

- thorough peer review by experienced researchers in your field

- rapid publication on acceptance

- support for research data, including large and complex data types

- gold Open Access which fosters wider collaboration and increased citations

- maximum visibility for your research: over 100M website views per year

At BMC, research is always in progress.

Learn more biomedcentral.com/submissions 